\title{
The Lorenz system and its generalizations as dynamo models with memory
}

\author{
Gleb Vodinchar ${ }^{1,3, *}$ and Evgeny Kazakov ${ }^{1,2}$ \\ ${ }^{1}$ Institute of Cosmophysical Researche and Radio Wave Propagation FEB RAS, \\ 684034 Paratunka, Kamchatka region, Russia \\ ${ }^{2}$ Kamchatka State Technical University, \\ 683003 Petropavlovsk-Kamchatsky, Russia \\ ${ }^{3}$ Vitus Bering Kamchatka State University, \\ 683032 Petropavlovsk-Kamchatsky, Russia
}

\begin{abstract}
The one of the known applications of the classical Lorenz system is an axisymmetric $\alpha \omega$-dynamo with a dynamical quenching of the $\alpha$-effect by the helicity. In this paper we consider generalizations of the Lorentz system, which are the models of $\alpha^{2}$ - and $\alpha^{2} \omega$-dynamo. The cases of finite and infinite memory in the quenching functional are considered. The conditions for the existence of stationary dynamo regimes and regimes of regular and chaotic inversions are analytically and numerically studded.
\end{abstract}

\section{Introduction}

It is well known that in real cosmic dynamo-systems an reversal phenomenon is observed, and the series of reversals contain both regular and chaotic components [1,2]. The modern models of magneto-convection in the cores of planets and convective zones of stars successfully reproduce certain features of reversals, but direct simulation in time scales comparable to the time of the existence of celestial bodies is impossible. In this case, the mean-field models and the concept of the alpha-effect become indispensable. These models make it possible to obtain long-term realizations of the field dynamics, but the solutions depend very much on the predetermined spatial structure of the alpha-effect in both scalar and anisotropic cases [3-5].

Therefore, interesting extremely simplified dynamo models are interesting in which the spatial structure of the fields is not predetermined and chaotic inversions arise. We have in mind are completely deterministic models, without parameter fluctuations or noises. Such models are dynamical systems of small dimension and describe only the basic phenomenology of dynamo - mutual generation of two field components by alpha effect and (possibly) differential rotation, as well as nonlinear quenching of generation. The such systems primarily include the Rikitake system [6] in which chaotic reversal arise, but this system is laboratory, its derivation from the MHD equations is very problematic. Another example of a classical system with stationary regimes and regimes of regular and chaotic reversals is the classical Lorentz system [7]. The Lorentz system as a dynamo model was proposed by [8] for explanation of the chaotic component of the solar cycle.

\footnotetext{
*e-mail: gvodinchar@ikir.ru
} 
In the present paper we consider some generalizations of the Lorenz system, as the simplest models of the mean-field dynamo, in which the spatial structure of the fields is not taken into account.

\section{Model equations}

Generation of an axisymmetric magnetic mean field $\mathbf{B}$ can be described by the equation

$$
\frac{\partial \mathbf{B}}{\partial t}=\nabla \times(\mathbf{v} \times \mathbf{B})+\nabla \times(\alpha \mathbf{B})+\eta \nabla^{2} \mathbf{B}
$$

where $\mathbf{v}$ - predetermined toroidal velocity field (usually this differential rotation), $\alpha$-effect is responsible for field generation by turbulence, $\eta$ - turbulent magnetic diffusivity. We assume that the feedback is manifested in a predetermined dependence $\alpha=\alpha_{0}(\mathbf{r})-F\left(H_{B}\right)$, where $\alpha_{0}$ - value of alpha-effect in the absence of the magnetic field, and $F\left(H_{B}\right)$ - is a convolution integral from the present and previous values of the magnetic helicity $H_{B}$. This predetermined expression $F\left(H_{B}\right)$ are specify the model of feedback - dynamical quenching of $\alpha$-effect by helicity. Note that we assume convolution in $F\left(H_{B}\right)$ only by time, and not by spatial coordinates. This means that in our model there is memory, but not spatial non-locality.

We believe that the spatial structure of the field is simplest and can be described by a one poloidal and a one toroidal modes. Thus we consider only the largest-scale structure of the mean field. This approximation has the form

$$
\mathbf{B}=B^{T}(t) \mathbf{b}^{T}(\mathbf{r})+B^{P}(t) \mathbf{b}^{P}(\mathbf{r}),
$$

where the energies of both magnetic modes are equal to one. In this case, the helicity of the field

$$
H_{B}=\int \mathbf{B}(\nabla \times \mathbf{B}) d \mathbf{r}=B^{T}(t) B^{P}(t) \int\left[\mathbf{b}^{T}\left(\nabla \times \mathbf{b}^{P}\right)+\mathbf{b}^{P}\left(\nabla \times \mathbf{b}^{T}\right)\right] d \mathbf{r} \sim B^{T}(t) B^{P}(t)
$$

Then, the evolution of field be determined by the behavior of scalar amplitudes $B^{T}(t)$ and $B^{P}(t)$ for which the following equations are valid:

$$
\begin{aligned}
& \frac{d B^{T}}{d t}=\left(\omega+\xi\left(\alpha_{0}-F\right)\right) B^{P}-\sigma B^{T}, \\
& \frac{d B^{P}}{d t}=\left(\alpha_{0}-F\right) B^{T}-B^{P}, \\
& F=\int_{0}^{t} J(t-\tau) B^{T}(\tau) B^{P}(\tau) d \tau,
\end{aligned}
$$

where $\sigma$ - ratio of diffusion times of $\mathbf{b}^{T}$ and $\mathbf{b}^{P}, \xi$ - ratio of efficiencies of the $\alpha$-effect for the poloidal and toroidal components, and $J(t)-$ is a sufficiently arbitrary kernel with the property that $J(+\infty)=0$.

These dimensionless equations can be obtained from $(1,2)$ using the Galerkin method. The timescale in (3) - is the $\mathbf{b}^{P}$ (r) diffusion time. It is known, that if we take the one toroidal and one poloidal modes of free decay of a magnetic field with the same spatial scales, then the poloidal mode has an eigenvalue smaller. Therefore, in the future, we believe that $\sigma>1$.

The integro-differential system (3) is a simplest model of the $\alpha^{2} \omega$-dynamo with dynamical quenching. In particular, for $\xi=0$ it describes a $\alpha \omega$-dynamo, and for $\omega=0$ it describes a $\alpha^{2}$-dynamo. 
We introduce the parameters $s=\left(\omega+\xi \alpha_{0}\right) / \sigma>0$ and $D=s \alpha_{0}>0$, and replace the variables:

$$
x(t)=B^{T}(t), \quad y(t)=s B^{P}(t), \quad z(t)=s F(t)
$$

Then system (3) can be written in the form

$$
\begin{aligned}
& \frac{d x}{d t}=\left(\sigma-\frac{\xi}{s^{2}} z\right) y-\sigma x, \\
& \frac{d y}{d t}=(D-z) x-y, \\
& z(t)=\int_{0}^{t} J(t-\tau) x(\tau) y(\tau) d \tau .
\end{aligned}
$$

We note that $s^{2} \sigma \geq \xi D$, since $\omega \geq 0$. Therefore $s^{2} \sigma=\xi D$ corresponds to the $\omega=0$, that is $\alpha^{2}$-dynamo.

Thus, the system (5) describes:

- $\alpha^{2} \omega$-dynamo, if $\xi \neq 0$ and $s^{2} \sigma>\xi D$;

- $\alpha^{2}$-dynamo, if $\xi \neq 0$ and $s^{2} \sigma=\xi D$;

- $\alpha \omega$-dynamo, if $\xi=0$.

It is clear that this system has a single stationary point $(0,0,0)$. This point will be stable for $D<1$, and unstable for $D>1$. Therefore, $D$ is a dynamo-number.

Formally, the integral term in (5) is a sign of memory (hereditarity) in this model. At the same time, it can be excluded for certain types of nuclei with exponential asymptotics $\sim e^{-b t}$, $b>0$, that's the true hereditarity in these cases is not. The reason is that the kernels with exponential asymptotics are integrable on the entire numerical axis and we can talk about the finite duration of the memory $T \sim \int_{0}^{+\infty} J(t) d t \sim 1 / b$. Then the memory effects will not be manifested on time scales greater than $T$.

If the kernel is not integrable on the time axis, the memory in the model has an unlimited duration, so we can talk about true hereditarity. For example, this will be for a power kernel $J(t)=(1+t)^{-\alpha}, 0<\alpha \leq 1$. In this case, the integral term in the system (5) can not be eliminated. Also, kernels of the form $J(t)=t^{n} e^{-b t}, n=1,2,3, \ldots$ are of some interest. In this case, we can speak of a delay in quenching, since the quenching functional depends little on the values of the field components at instants close to the present time.

Let us now consider how the integral term is eliminated from (5) for some types of kernels with exponential asymptotics. Assume that the kernel $J(t)$ be a solution of the following Cauchy problem for a linear homogeneous equation $n$-th order with constant coefficients:

$$
\begin{aligned}
& a_{n} \frac{d^{n} J(t)}{d t^{n}}+a_{n-1} \frac{d^{n-1} J(t)}{d t^{n-1}}+\cdots+a_{0} J(t)=0, \\
& J(0)=q_{0}, \quad J^{(1)}(0)=q_{1}, \quad \ldots \quad J^{(n-1)}(0)=q_{n-1} .
\end{aligned}
$$

Differentiate third equation of system (5) $k$ times in a row, we obtain:

$$
\frac{d^{k} z}{d t^{k}}=\int_{0}^{t} J^{(k)}(t-\tau) x(\tau) y(\tau) d \tau+\sum_{l=0}^{k-1} J^{(l)}(0) \frac{d^{k-1-l}}{d t^{k-1-l}} x(t) y(t),
$$


We multiply (7) by $a_{k}$ and sum up by $k$ from 0 to $n$, taking into account (6):

$$
\begin{aligned}
& \sum_{k=0}^{n} a_{k} \frac{d^{k} z}{d t^{k}}=\sum_{k=0}^{n} a_{k} \sum_{l=0}^{k-1} q_{l} \frac{d^{k-1-l}}{d t^{k-1-l}} x(t) y(t), \\
& z^{(k)}(0)=\sum_{l=0}^{k-1} q_{l} x^{(k-1-l)}(0) y^{(k-1-l)}(0), \quad k=0,1, \ldots, n-1 .
\end{aligned}
$$

So, in this case, the third equation of the system (5) is equivalent to the equations (8). The integro-differential system reduces to a differential system of order $n+2$ with initial conditions on $n$ phase variables.

\section{Exponential kernel}

Now consider the important case of an exponential kernel $J(t)=e^{-b t}, b>0$. Then the equation $(6,8)$ takes the form:

$$
\frac{d J(t)}{d t}+b J(t)=0, \quad J(0)=1
$$

and

$$
\frac{d z(t)}{d t}=-b z(t)+x(t) y(t), \quad z(0)=0,
$$

respectively.

Thus, if the kernel is exponential, then system the (5) can be written in the form

$$
\begin{aligned}
& \frac{d x}{d t}=\left(\sigma-\frac{\xi}{s^{2}} z\right) y-\sigma x, \\
& \frac{d y}{d t}=(D-z) x-y, \\
& \frac{d z}{d t}=q(x(t), y(t))-b z, \\
& z(0)=0 .
\end{aligned}
$$

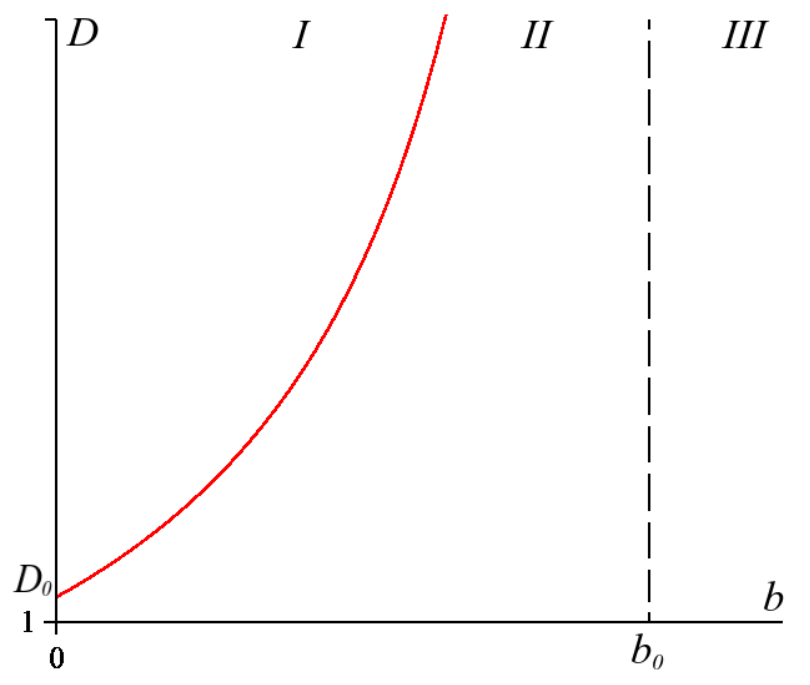

Figure 1. Areas of different regimes in the $(b, D)$-plane. I - regime with reversals, II and III - stationary regime of dynamo. $D_{0}(\sigma, s)>1$ and $b_{0}=\sigma-1>0$, if $\sigma>1$. Red line is half-hyperbola, and the dotted line is its asymptote. 
In particular, for $\xi=0$ and $q(x, y)=x y$, the system (9) takes the form a classical Lorenz system [7]. Therefore, we can speak of a system (5), which describes various dynamo mechanisms, as about broad generalization of the Lorentz system.

From this point of view, the Lorenz system itself is a simplest $\alpha \omega$-dynamo model, when the helicity with an exponential kernel is quenched the alpha effect.

\subsection{Lorenz system as $\alpha \omega$-dynamo model}

We consider the system (9) for $\xi=0$ and without the condition $z(0)=0$. It is well known that it has two symmetric stationary points in addition to the zero stationary point:

$$
( \pm \sqrt{b(D-1)}, \pm \sqrt{b(D-1)}, D-1)
$$

At these points the third coordinates are nonzero, if $D \neq 1$. Therefore, they are the stationary points of the dynamical system (without condition $z(0)=0$ ), but they are "punctured" in the phase space of the dynamo model (9). However, the transitions of the phase trajectories between these "punctured" points signify an reversal of the field. If these points are asymptotically stable, then the approximation of the phase trajectory to one of them corresponds to the stationary regime of the dynamo. It is also clear that the symmetry $x(t) \leftrightarrow y(t)$ of the system (9) provides the identical structure of the phase space in the vicinity of these points.

It is easy to get areas of dynamic regimes shown in Fig. 1. Of course, the dynamics of this system are well known. However, in numerous studies is usually varied the parameter, which we have designated as $D$. The $\sigma$ and $b$ is fixed: $\sigma=10$ and $b=8 / 3$. Such values are due to the problem of convection in a plane layer, from which the Lorenz system arose [7]. In a dynamo problem $\sigma>1$, but the characteristic times of dissipation poloidal and toroidal
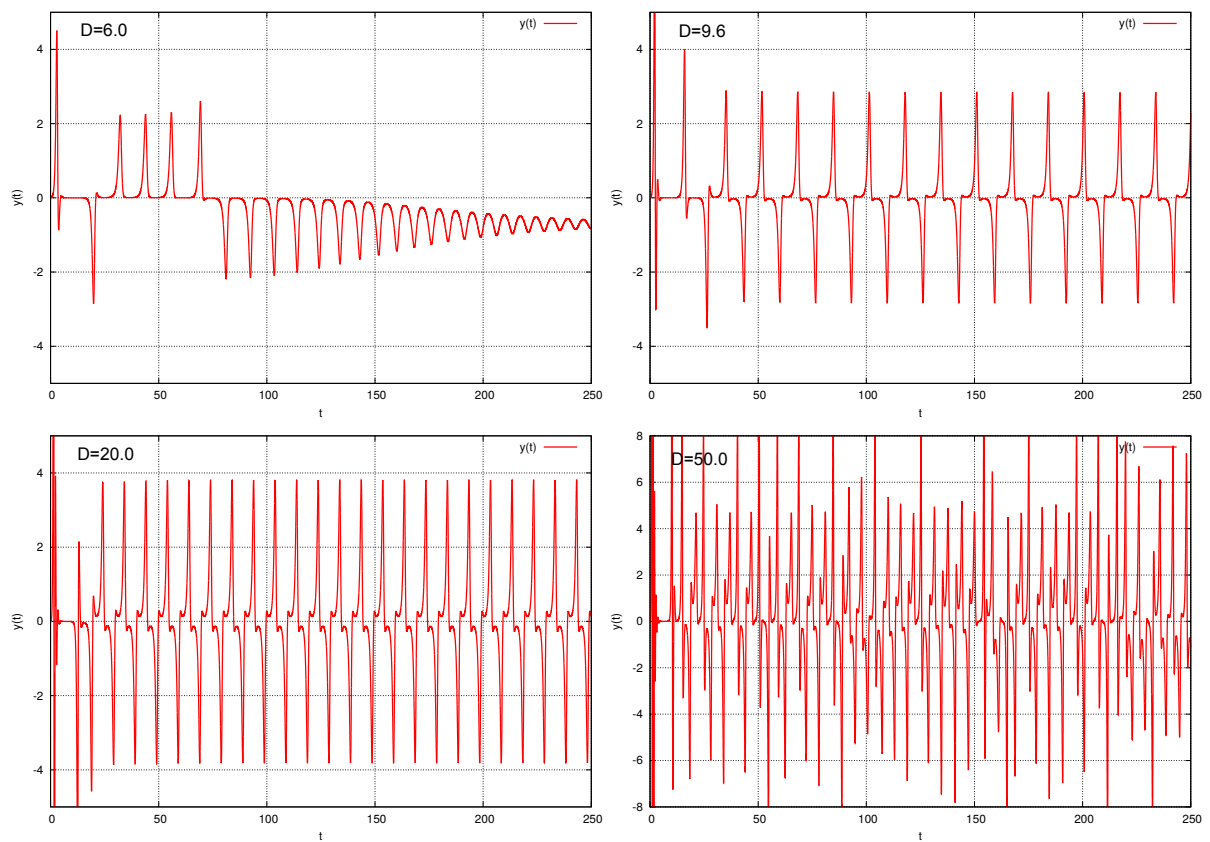

Figure 2. The solutions for the $\alpha \omega$-dynamo at $b=0.1$ and different $D$. 

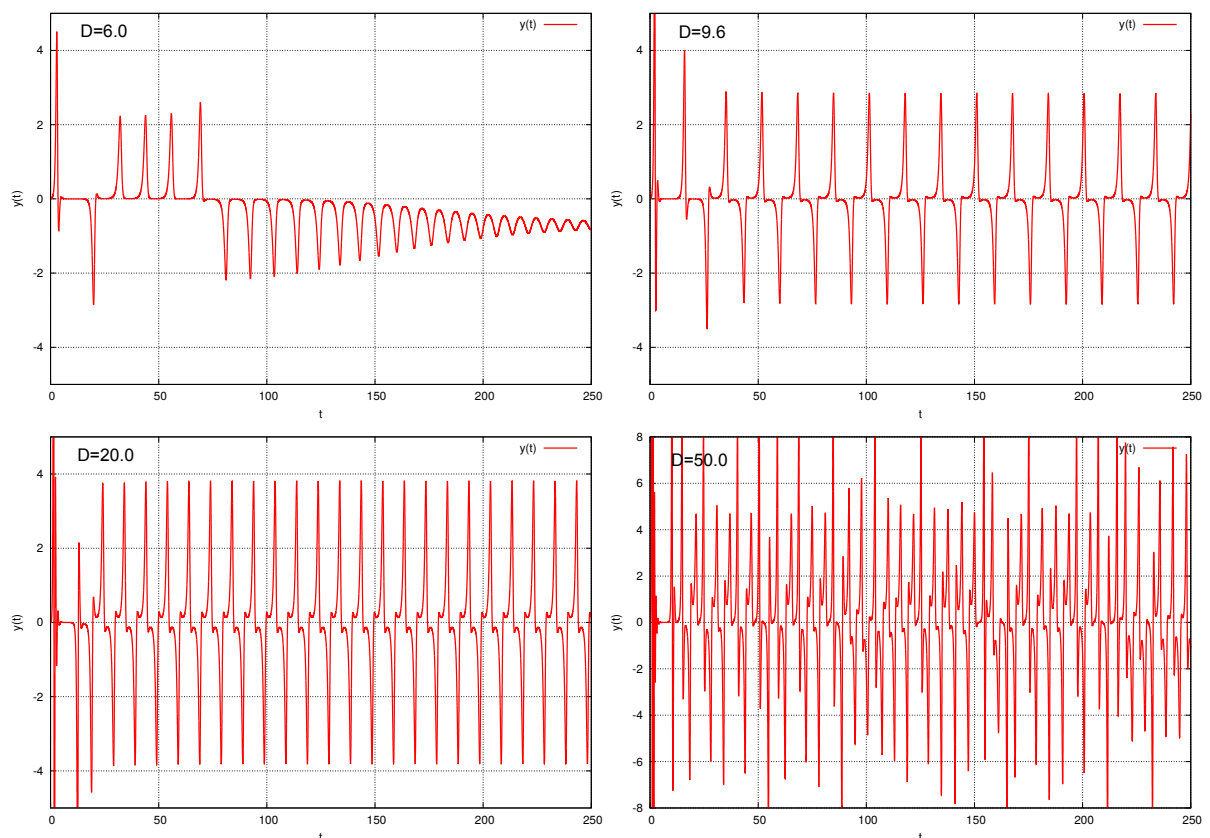

Figure 3. The solutions for the $\alpha \omega$-dynamo at $b=0.1$ and different $D$.

components do not differ by an order. Thus, for the classical two-mode Parker dynamo [9], the ratio of the eigenvalues of the modes gives $\sigma=3.37$. The parameter $b$ in our problem determines the effective memory duration and should be varied.

From Fig. 1 that for the reversal regime to appear it is necessary to have sufficiently large values of the dynamo number and a sufficiently long memory. Easy to establish that $D_{0}=\sigma(\sigma+3) /(\sigma+1)$. This expression reaches the least value equal to 9 for $\sigma=3$, which is characteristic of the Parker dynamo. The asymptote $b_{0}=\sigma-1$, then for reversals it is necessary $T \gtrsim 0.5$.

The numerical simulation of the Lorenz system for $\sigma=3.37$, and $b=0.5,1.0,5.0$ gave phase trajectories, which qualitatively not differing from the well-known ones in the classical case $\sigma=10$, and $b=8 / 3$. However, for $b=0.1$, we obtained the regimes shown in Fig. 2. In this case the points (11) lose stability when $D=9.6$. It can be seen that the solutions have the form of dynamo-bursts, and with an increase in $D$ the regular bursts are replaced by chaotic.

\section{$3.2 \alpha^{2}$ - and $\alpha^{2} \omega$-dynamo}

Suppose now that in the system (9) $\xi \neq 0$ and $s^{2} \sigma=\xi D$. In this case system describe $\alpha^{2}$ dynamo. When a dynamo is working (i.e. $D>1$ ) and without the condition $z(0)=0$, system has a one pair of nonzero stationary points:

$$
( \pm \sqrt{b(\sqrt{D}-1)}, \pm \sqrt{b(D-\sqrt{D})}, D-\sqrt{D}) .
$$

As in the previous case, these points themselves are "punctured" from the phase space of the dynamo model, but their stability corresponds to the regime of the stationary dynamo. 

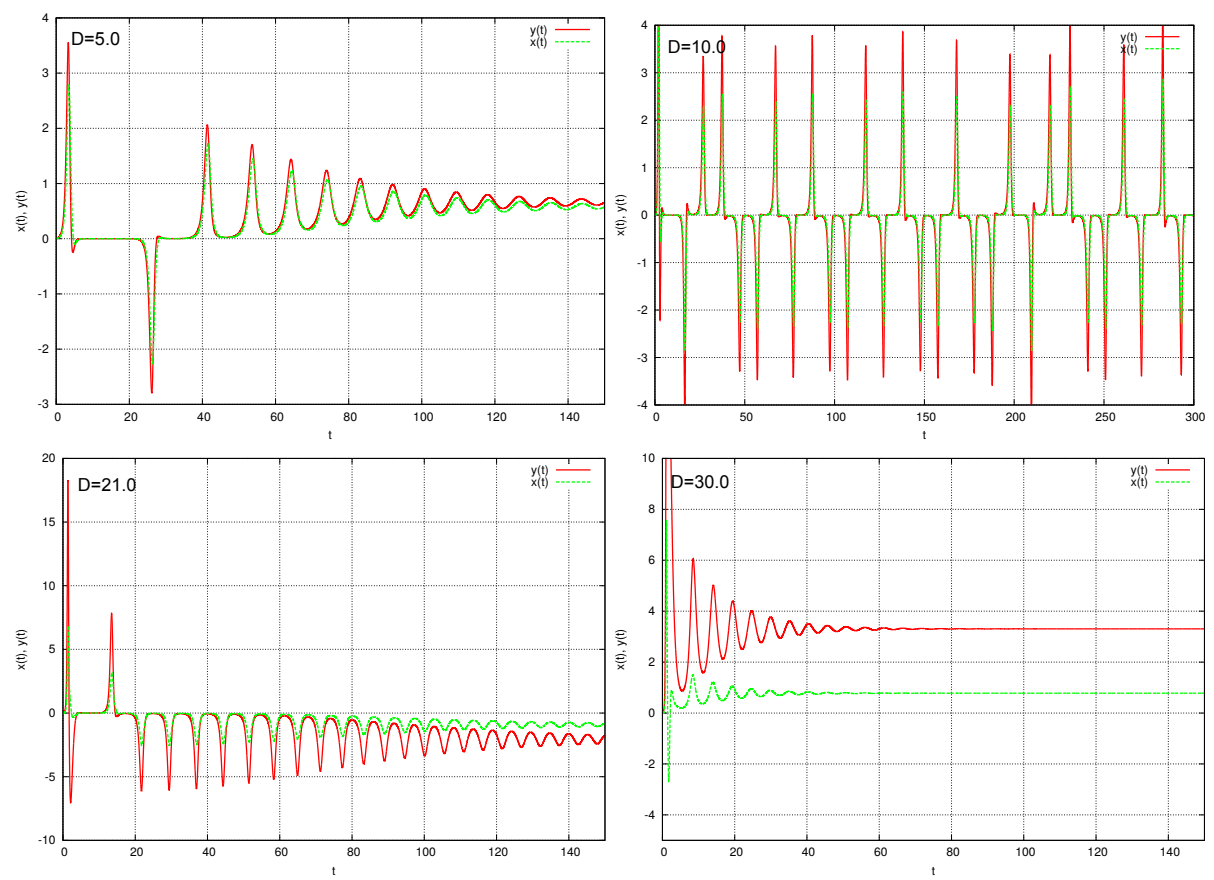

Figure 4. The solutions for the $\alpha^{2} \omega$-dynamo with quenching by helicity, $s=1$, and $b=0.1$.

An analytic study of the stability conditions of these points is very difficult, so it was carried out with the help of numerous computational experiments at various parameters. It turned out that these points are always stable. Thus, reversals in our model $\alpha^{2}$-dynamos do not arise.

It is known that a model of $\alpha^{2}$-dynamo for scalar $\alpha$-effect have difficulties to explain the reversals. However, there are series of works in which it is shown that for a some special radial inhomogeneity of the $\alpha$-effect, reversals in $\alpha^{2}$-dynamo can arise [3-5]. Therefore, we can say that in order to reproduce the reversal phenomenon in $\alpha^{2}$-dynamo, it is necessary to take into account spatial inhomogeneities. In our simplest model, the spatial structure of the fields is not used in any way. This is the reason for the absence of reversal regimes.

The system (9) describes the mechanism $\alpha^{2} \omega$-dynamo, if $\xi \neq 0$ and ${ }^{2} \sigma>\xi D$. As in the previous case, with such values of the parameters and $D>1$ the system there are a pair of nonzero symmetric stationary points. It is self-evident that we did not use conditions $z(0)=0$. The expressions for the coordinates of these points can be obtained analytically in an explicit form, but they very cumbersomeness and we are not write this formulas. The stability of points can also be checked only by numerical methods.

The numerical simulation showed that in such a model the stationary regimes and the regimes of regular and chaotic reversals can arise under. If $b$ is small, dynamo-bursts are arise (Fig. 3 for $b=0.1$ ). The examples of solutions for this model for $b=0.5$ are shown in Fig. 4. Here, bursts are no longer observed. It should be noted an interesting feature of the solutions for this model. If we rewrite inequality ${ }^{2} \sigma>\xi D$ in the form $D<\sigma s^{2} / \xi$, then the dynamo-number is bounded above for fixed $\sigma, s$, and $\xi$. It turns out that large values $D$ can stabilize the dynamo. This situation is typical. With the growth of the dynamo-number, the 

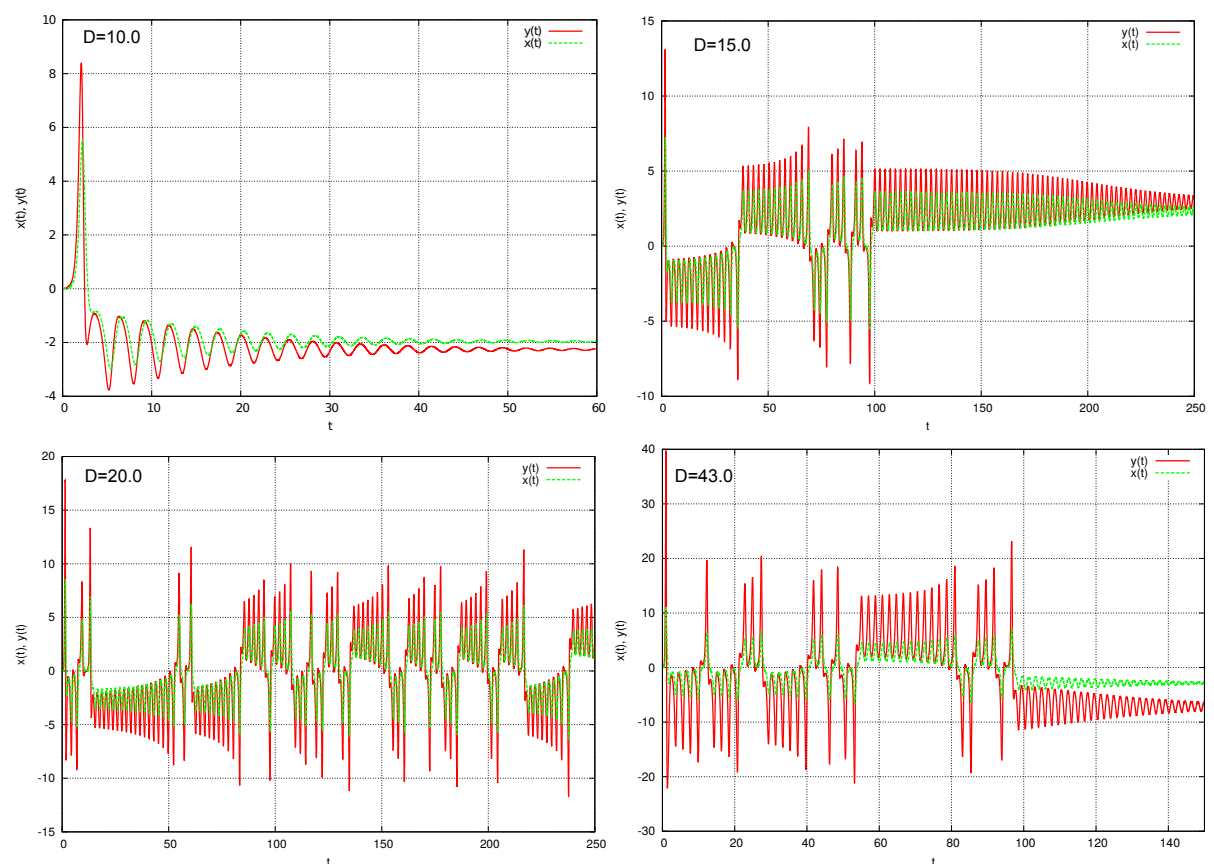

Figure 5. The solutions for the $\alpha^{2} \omega$-dynamo with quenching by helicity, $s=1$, and $b=0.5$.

stationary regime is first observed, then regular and/or chaotic reversals arise, then again the stationary regime.

\section{Power kernel}

We mentioned above that a sufficiently long memory is necessary for the appearance of an reversals in the case of exponential kernels. Then it can be assumed that the reversals always will be, for nonintegrable kernels with a power asymptotic and for large values of the dynamo number.

However, the simulation results suggest the opposite effect. An infinitely long memory leads the dynamo to a stationary regime. Some results are shown in fig. 4 . It can be seen that the dynamo stabilized. This happens even when the chaotic reversals regime first existed.

Therefore, we can conclude that for the reversals regime in the models described, a sufficiently long but finite memory is necessary.

\section{Conclusion}

- In the proposed models, the main dynamo regimes are realized - stationary, reversals (chaotic and regular), dynamo-bursts.

- In the «pure» $\alpha^{2}$-dynamo reproduction of reversals is impossible. Most likely the reason is that in our models there is no heterogeneity in the spatial distribution.

- For the occurrence of reversals requires a long, but finite memory. 

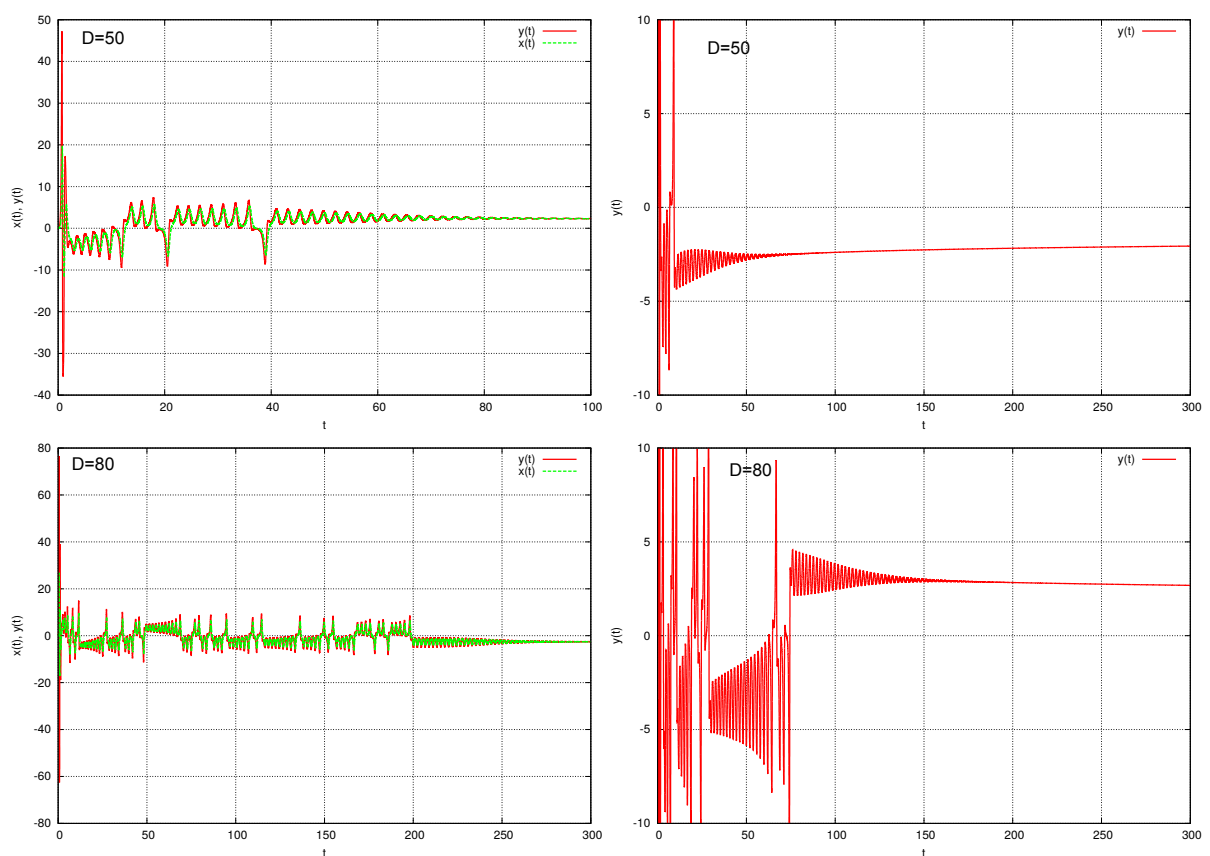

Figure 6. The solutions for the power kernel $J(t)=(1+t)^{-0.8} \cdot \alpha \omega$-dynamo on left panel and $\alpha^{2} \omega$ dynamo $(\xi=0.5)$ on right panel.

\section{Acknowledgement}

This work is supported by Russian Science Foundation (project 14-11-00194).

\section{References}

[1] R. T. Merril, M. W. McElhinny, and P. L. McFadden, The Magnetic Field of the Earth:

Paleomagnetism, the Core, and the Deep Mantle (Academic Press, London, 1996).

[2] M. Stix, The Sun. An Introduction (Springer, Berlin, 2002).

[3] D. R. Fearn, M. M. Rahman, Geophys. Astrophys. Fluid Dynam., 98:5, 385-406 (2004).

[4] A. Giesecke, G. Rüdiger, D. Elstner, Astron. Nachr., 326:8, 693-700 (2005).

[5] F. Stefani, G. Gerbeth, Phys. Rev. E, 67, 027302 (2003).

[6] T. Rikitake, Proc. Cambridge Philos. Soc., 54, 89-105 (1958).

[7] E. Lorenz, J. Atmos. Sci., 20, 130-141 (1963).

[8] A. A. Ruzmaikin, Comments on Astrophys., 9, 85-96 (1981).

[9] E. N. Parker, Astrophys. J., 122, 293-314 (1955). 